\title{
Equal-Gain Combining with Unequal Energy Constellations
}

\author{
Ranjan K. Mallik, Senior Member, IEEE, and George K. Karagiannidis, Senior Member, IEEE
}

\begin{abstract}
To compute the optimal bias in equal-gain combining (EGC) receivers with unequal energy constellations such as $M$-ary pulse amplitude modulation $(M$-PAM) and $M$-ary square quadrature amplitude modulation ( $M$-QAM), the estimation of the instantaneous fading gain magnitude in each diversity branch is needed, thus defeating the usefulness of EGC. In this paper, we present an exact analytical equation, which can be efficiently used to compute a suboptimal value for the bias, both for $M$-PAM and $M$-QAM constellations. The proposed suboptimal bias minimizes the symbol error probability, and depends on the statistics of the fading gain magnitudes and not on their instantaneous values. For the case of large number of branches, where the central limit theorem can be applied, the proposed equation for the computation of the suboptimal bias is derived in closed-form. Moreover, an approximate simple closed-form expression for the suboptimal bias is obtained, suitable for the high signal-to-noise ratio region. Numerical results verify the correctness and the accuracy of the proposed analytical formulation.
\end{abstract}

Index Terms-Equal-gain combining, $M$-ary pulse amplitude modulation ( $M$-PAM), $M$-ary square quadrature amplitude modulation ( $M$-QAM), suboptimal bias, symbol error probability, unequal energy signaling.

\section{INTRODUCTION}

A MONG the well known diversity techniques used in wireless communication systems to mitigate signal impairments due to fading, predetection equal-gain combining (EGC), often simply called EGC, is of great practical interest because it provides an intermediate solution as far as performance and implementation complexity are concerned. In predetection EGC, each diversity branch is weighted with the same factor, irrespective of the signal amplitude. Moreover, cophasing of all branch signals is needed to avoid signal cancellation. There are several approaches to the performance analysis of predetection EGC receivers both for independent and correlated fading channels (as in [1]-[7] and references therein). A comprehensive summary of most of the related work is included in [8]. However, in practice, EGC is useful for modulation schemes having equal energy symbols, like $M$-ary phase-shift keying ( $M$-PSK). With signaling schemes

Manuscript received July 12, 2005; revised March 17, 2006; accepted August 2, 2006. The associate editor coordinating the review of this paper and approving it for publication was C. Tellambura. The work of George K. Karagiannidis was supported in part by the Greek General Secretariat for Research and Development (GSRT) under the Greece-Montenegro Bilateral S\&T Cooperation Program (2006-2008).

R. K. Mallik is with the Department of Electrical Engineering, Indian Institute of Technology - Delhi, Hauz Khas, New Delhi 110016, India (email: rkmallik@ee.iitd.ernet.in).

G. K. Karagiannidis is with the Department of Electrical and Computer Engineering, Aristotle University of Thessaloniki, Thessaloniki 54 124, Greece (e-mail: geokarag@auth.gr).

Digital Object Identifier 10.1109/TWC.2007.05480. of unequal energy symbols, the instantaneous channel gain magnitudes of all branches are required to compute the optimal bias, thus defeating the advantage of EGC of avoiding channel estimation. In order to overcome this problem, we present an analytical approach for the evaluation of a suboptimal value for the bias, both for $M$-ary pulse amplitude modulation ( $M$-PAM) and $M$-ary square quadrature amplitude modulation ( $M$-QAM), which have unequal energy constellations. This suboptimal value minimizes the symbol error probability (SEP), and depends on the statistics of the fading gains and not on their instantaneous values. For the important case of large number of branches, where the central limit theorem can be applied, the proposed equation for the computation of the suboptimal bias is given in closed-form. Moreover, we obtain an approximate simple closed-form expression for the suboptimal bias, suitable for the high signal-to-noise ratio (SNR) region. Finally, we present numerical results to verify the correctness of the proposed analytical formulation.

The remainder of this paper is organized as follows. Section II presents the system model under consideration. In Section III, the conditional SEP in terms of the bias is derived, while in Sections IV and V suboptimal biases for $M$-PAM and $M$-QAM, respectively, are proposed. Subsequently, in Section VI, selected numerical results are presented. Some useful concluding remarks are finally provided in Section VII.

\section{System Model}

Consider a diversity reception system with $L$ branches in flat fading. The received complex baseband signal for the $k$ th diversity branch in a symbol duration $T_{s}$ is given by

$$
r_{k}(t)=\alpha_{k} e^{-\jmath \phi_{k}} s(t)+n_{k}(t), \quad k=1, \ldots, L, \quad 0 \leq t<T_{s},
$$

where $s(t)$ is the information bearing signal, $\alpha_{k}$ is the random magnitude and $\phi_{k}$ the random phase of the fading gain of the $k$ th branch, and $n_{k}(t)$, the additive noise, is a zeromean complex circular Gaussian random process with power spectral density $2 N_{0 k}$. The signal $s(t)$ is one of $M$ signaling waveforms $s_{0}(t), \ldots, s_{M-1}(t)$, each having support $\left[0, T_{s}\right)$, with

$$
\int_{0}^{T_{s}}\left|s_{i}(t)\right|^{2} d t=2 E_{s_{i}}, \quad i=0, \ldots, M-1 .
$$

Moreover, the processes $n_{1}(t), \ldots, n_{L}(t)$ are independent of each other and the random fading gains $\alpha_{1} e^{-\jmath \phi_{1}}, \ldots, \alpha_{L} e^{\jmath \phi_{L}}$ are independent of $n_{1}(t), \ldots, n_{L}(t)$.

Consider $M$-PAM signaling, $M$ being an even number, in which

$$
s_{i}(t)=\frac{(2 i-M+1) d}{2} p(t), \quad i=0, \ldots, M-1,
$$


where $p(t)$ is a baseband pulse (can be real or complex) with support $\left[0, T_{s}\right)$ and satisfying

$$
\int_{0}^{T_{s}}|p(t)|^{2} d t=1
$$

Combining (2) and (3), we have

$$
E_{s_{i}}=\frac{(2 i-M+1)^{2} d^{2}}{8}
$$

Assuming equiprobable signaling, the average signal energy $E_{s, a v}$ is given by

$$
E_{s, a v}=\frac{1}{M} \sum_{i=0}^{M-1} E_{s_{i}}=\frac{\left(M^{2}-1\right) d^{2}}{24} .
$$

Consider the projection of the signals on $p(t)$, which corresponds to the axis of a one-dimensional coordinate system. Denoting

$$
\begin{aligned}
& r_{k}=\int_{0}^{T_{s}} r_{k}(t) p^{*}(t) d t, \quad s=\int_{0}^{T_{s}} s(t) p^{*}(t) d t, \\
& n_{k}=\int_{0}^{T_{s}} n_{k}(t) p^{*}(t) d t,
\end{aligned}
$$

we get from (1)

$$
r_{k}=\alpha_{k} e^{-\jmath \phi_{k}} s+n_{k}, \quad k=1, \ldots, L,
$$

where $n_{1}, \ldots, n_{L}$ are independent complex Gaussian random variables, $n_{k}$ having a $\mathcal{C N}\left(0,2 N_{0 k}\right)$ distribution, and $s$ is one of $M$ signal points $s_{0}, \ldots, s_{M-1}$, such that, from (3)

$$
s_{i}=\frac{(2 i-M+1) d}{2}, \quad i=0, \ldots, M-1 .
$$

Note that $s_{i}$ is a real number.

When EGC is used, the decision variable corresponding to the $i$ th signal is given by

$$
\begin{aligned}
D_{i} & =\operatorname{Re}\left\{s_{i}^{*} \sum_{k=1}^{L} \frac{e^{\jmath \phi_{k}}}{\sqrt{N_{0 k}}} r_{k}\right\}-B_{i} \\
& =s_{i} \operatorname{Re}\left\{\sum_{k=1}^{L} \frac{e^{\jmath \phi_{k}}}{\sqrt{N_{0 k}}} r_{k}\right\}-B_{i},
\end{aligned}
$$

where $B_{i}$ is the bias. If signal $s_{j}(t)$ is transmitted, the receiver makes the decision

$$
\hat{j}=\arg \left\{\max _{0 \leq i \leq M-1} D_{i}\right\} .
$$

The optimal bias, which minimizes the SEP conditioned on $\alpha_{1} e^{-\jmath \phi_{1}}, \ldots, \alpha_{L} e^{\jmath \phi_{L}}$, is given by

$$
\begin{aligned}
B_{i, o p t} & =\frac{\left|s_{i}\right|^{2}}{2} \sum_{k=1}^{L} \frac{\alpha_{k}}{\sqrt{N_{0 k}}} \\
& =E_{s_{i}} \sum_{k=1}^{L} \frac{\alpha_{k}}{\sqrt{N_{0 k}}} \\
& =\frac{(2 i-M+1)^{2} d^{2}}{8} \sum_{k=1}^{L} \frac{\alpha_{k}}{\sqrt{N_{0 k}}} .
\end{aligned}
$$

To compute the optimal bias, we need the estimation of the fading gain magnitudes $\alpha_{1}, \ldots, \alpha_{L}$, which defeats the purpose of EGC. Hence we try to get a suboptimal bias $B_{i}$ which depends on the channel statistics, but not on the instantaneous values of $\alpha_{1}, \ldots, \alpha_{L}$.

We express the suboptimal bias as

$$
B_{i}=\frac{\left|s_{i}\right|^{2}}{2} A=E_{s_{i}} A=\frac{(2 i-M+1)^{2} d^{2}}{8} A .
$$

From (10), the decision boundary between the signal points $s_{i}$ and $s_{i+1}$ is given by a point with abscissa $X_{i}$ which satisfies

$$
s_{i} X_{i}-B_{i}=s_{i+1} X_{i}-B_{i+1}, \quad i=0, \ldots, M-2 .
$$

This implies (from (14) and (9))

$$
\begin{aligned}
X_{i} & =\frac{B_{i+1}-B_{i}}{s_{i+1}-s_{i}} \\
& =\left(\frac{s_{i+1}+s_{i}}{2}\right) A \\
& =\frac{(2 i-M+2)}{2} A d .
\end{aligned}
$$

\section{Symbol ERror Probability}

When $s_{i}(t)$ is transmitted, we have from (8) and (10)

$$
\begin{aligned}
\left.\operatorname{Re}\left\{\sum_{k=1}^{L} \frac{e^{\jmath \phi_{k}}}{\sqrt{N_{0 k}}} r_{k}\right\}\right|_{s=s_{i}}= & s_{i} \sum_{k=1}^{L} \frac{\alpha_{k}}{\sqrt{N_{0 k}}} \\
& +\operatorname{Re}\left\{\sum_{k=1}^{L} \frac{e^{\jmath \phi_{k}}}{\sqrt{N_{0 k}}} n_{k}\right\} \\
= & s_{i} \sum_{k=1}^{L} \frac{\alpha_{k}}{\sqrt{N_{0 k}}}+w
\end{aligned}
$$

where $w$ has a $\mathcal{N}(0, L)$ distribution.

For $i=1, \ldots, M-2$, the probability of correct decision (PCD), given $s_{i}$ is transmitted, can be expressed as

$$
P_{c_{i}}=\operatorname{Pr}\left[X_{i-1}<s_{i} \sum_{k=1}^{L} \frac{\alpha_{k}}{\sqrt{N_{0 k}}}+w<X_{i}\right] .
$$

Substituting (9) and (15) in (17), we get

$$
\begin{aligned}
P_{c_{i}}=\operatorname{Pr} & {\left[\frac{(2 i-M)}{2} A d\right.} \\
& <\frac{(2 i-M+1) d}{2} \sum_{k=1}^{L} \frac{\alpha_{k}}{\sqrt{N_{0 k}}}+w \\
& \left.<\frac{(2 i-M+2)}{2} A d\right]
\end{aligned}
$$

From the statistics of $w$, we can express the conditional PCD when $s_{i}(t)$ is transmitted (which is a function of $A$ ), 
conditioned on $\alpha_{1}, \ldots, \alpha_{L}$, as

$$
\begin{aligned}
P_{c_{i}}\left(\left\{\alpha_{k}\right\}, A\right)=Q\left(\frac{d}{2 \sqrt{L}}[(2 i-M) A\right. \\
\left.\left.\quad-(2 i-M+1) \sum_{k=1}^{L} \frac{\alpha_{k}}{\sqrt{N_{0 k}}}\right]\right) \\
+Q\left(\frac { d } { 2 \sqrt { L } } \left[(2 i-M+1) \sum_{k=1}^{L} \frac{\alpha_{k}}{\sqrt{N_{0 k}}}\right.\right. \\
\quad-(2 i-M+2) A]) \\
i=1, \ldots, M-2 .
\end{aligned}
$$

It can be easily shown from symmetry of the signal points that

$$
P_{c_{i}}\left(\left\{\alpha_{k}\right\}, A\right)=P_{c_{M-1-i}}\left(\left\{\alpha_{k}\right\}, A\right), \quad i=0, \ldots, M-1 \text {. }
$$

Therefore, the conditional PCD, conditioned on $\left\{\alpha_{k}\right\}$, is given by

$$
\begin{aligned}
P_{c}\left(\left\{\alpha_{k}\right\}, A, M\right) & \frac{2}{M}\left[\sum _ { i = \frac { M } { 2 } } ^ { M - 2 } \left\{Q \left(\frac{d}{2 \sqrt{L}}[(2 i-M) A\right.\right.\right. \\
& \left.\left.-(2 i-M+1) \sum_{k=1}^{L} \frac{\alpha_{k}}{\sqrt{N_{0 k}}}\right]\right) \\
+ & Q\left(\frac { d } { 2 \sqrt { L } } \left[(2 i-M+1) \sum_{k=1}^{L} \frac{\alpha_{k}}{\sqrt{N_{0 k}}}\right.\right. \\
& -(2 i-M+2) A])\} \\
+ & Q\left(\frac{d}{2 \sqrt{L}}[(M-2) A\right. \\
& \left.\left.\left.-(M-1) \sum_{k=1}^{L} \frac{\alpha_{k}}{\sqrt{N_{0 k}}}\right]\right)\right] .
\end{aligned}
$$

The instantaneous SNR of the $k$ th branch is given by

$$
\gamma_{k}=\frac{E_{s, a v} \alpha_{k}^{2}}{N_{0 k}}
$$

For EGC, the instantaneous SNR of the combiner output can be expressed as

$$
\begin{aligned}
\gamma & =\frac{1}{L}\left(\sum_{k=1}^{L} \sqrt{\gamma_{k}}\right)^{2} \\
& =\frac{E_{s, a v}}{L}\left(\sum_{k=1}^{L} \frac{\alpha_{k}}{\sqrt{N_{0 k}}}\right)^{2} \\
& =\frac{\left(M^{2}-1\right) d^{2}}{24 L}\left(\sum_{k=1}^{L} \frac{\alpha_{k}}{\sqrt{N_{0 k}}}\right)^{2}
\end{aligned}
$$

Using the fact that $Q(x)=1-Q(-x)$ in (21), we can now express the conditional SEP, conditioned on $\gamma$, as

$$
\begin{aligned}
& P_{e}(\gamma, A, M)=\frac{2}{M}\left[\sum _ { i = \frac { M } { 2 } } ^ { M - 2 } \left\{Q \left(\sqrt{\frac{6}{M^{2}-1}}\right.\right.\right. \\
& \times[(2 i-M+1) \sqrt{\gamma} \\
& \left.\left.-(2 i-M) A \sqrt{\frac{E_{s, a v}}{L}}\right]\right) \\
& +Q\left(\sqrt{\frac{6}{M^{2}-1}}\right. \\
& \times\left[(2 i-M+2) A \sqrt{\frac{E_{s, a v}}{L}}\right. \\
& -(2 i-M+1) \sqrt{\gamma}])\} \\
& +Q\left(\sqrt{\frac{6}{M^{2}-1}}[(M-1) \sqrt{\gamma}\right. \\
& \left.\left.\left.-(M-2) A \sqrt{\frac{E_{s, a v}}{L}}\right]\right)\right] \text {. }
\end{aligned}
$$

It is clear from (24) that when

$$
A=A_{o p t}=\sum_{k=1}^{L} \frac{\alpha_{k}}{\sqrt{N_{0 k}}},
$$

we get the optimal conditional SEP, given by

$$
P_{e, o p t}(\gamma, M)=\frac{2(M-1)}{M} Q\left(\sqrt{\frac{6 \gamma}{M^{2}-1}}\right) \text {. }
$$

Let

$$
B=A \sqrt{\frac{E_{s, a v}}{L}}
$$

denote the normalized bias. In addition, let

$$
g=\frac{3}{M^{2}-1} .
$$

We can now denote $P_{e}(\gamma, A, M)$ in (24) as $P_{e}(\gamma, B, M)$, which is given by

$$
\begin{gathered}
P_{e}(\gamma, B, M)=\frac{2}{M}\left[\sum_{i=\frac{M}{2}}^{M-2}\{Q(\sqrt{2 g}[(2 i-M+1) \sqrt{\gamma}\right. \\
\quad-(2 i-M) B]) \\
+Q(\sqrt{2 g}[(2 i-M+2) B \\
+Q(\sqrt{2 g}[(M-1) \sqrt{\gamma} \\
+(M-2) B])]
\end{gathered}
$$




\section{Suboptimal BiAs For $M$-PAM}

Let $f_{\gamma}(\cdot)$ denote the probability density function (p.d.f.) of $\gamma$. To find a suboptimal value of $B$, which depends on the statistics of $\gamma$ and not on the instantaneous value of $\gamma$, we need to obtain a value of $B$ which minimizes the SEP averaged over $\gamma$, that is, we need to find $B_{\text {subpot }}$ given by

$$
B_{\text {subopt }}=\arg \left\{\min _{B>0} \int_{0}^{\infty} P_{e}(x, B, M) f_{\gamma}(x) d x\right\} .
$$

In order to estimate $f_{\gamma}(x)$ in (30), the Beaulieu infinite series approximation of the sum of independent random variables can be efficiently applied for Rayleigh and Nakagami- $m$ fading channels [1], [9]. Note that for the important practical case of $L=2$ with Rayleigh fading and unequal mean SNRs, $f_{\gamma}(x)$ can be expressed in closed-form [10]. In the next section, we also present a closed-form equation for the evaluation of $B$, when large $L$ is assumed.

Differentiating $P_{e}(\gamma, B, M)$ in (29) with respect to $B$, we get

$$
\begin{aligned}
\frac{\partial P_{e}(\gamma, B, M)}{\partial B}=- & \frac{2 \sqrt{g}}{M \sqrt{\pi}}\left[\sum_{i=\frac{M}{2}}^{M-2}\{-(2 i-M)\right. \\
& \times e^{-g((2 i-M+1) \sqrt{\gamma}-(2 i-M) B)^{2}} \\
+ & (2 i-M+2) \\
& \left.\times e^{-g((2 i-M+2) B-(2 i-M+1) \sqrt{\gamma})^{2}}\right\} \\
- & (M-2) \\
& \left.\times e^{-g((M-1) \sqrt{\gamma}-(M-2) B)^{2}}\right] .
\end{aligned}
$$

Now, applying Leibnitz rule [11] in (30) and using (31), $B_{\text {subopt }}$ can be calculated by solving numerically for $B$ the analytical single integral equation

$$
\begin{aligned}
& J(B) \\
& \triangleq \sum_{i=\frac{M}{2}}^{M-2}\{-(2 i-M) \\
& \quad \times \int_{0}^{\infty} e^{-g((2 i-M+1) \sqrt{x}-(2 i-M) B)^{2}} f_{\gamma}(x) d x \\
& +(2 i-M+2) \\
& \left.\quad \times \int_{0}^{\infty} e^{-g((2 i-M+2) B-(2 i-M+1) \sqrt{x})^{2}} f_{\gamma}(x) d x\right\} \\
& -(M-2) \\
& \quad \times \int_{0}^{\infty} e^{-g((M-1) \sqrt{x}-(M-2) B)^{2}} f_{\gamma}(x) d x \\
& =0 .
\end{aligned}
$$

We can obtain $B_{\text {subopt }}$ by applying the iterative NewtonRaphson method for root finding on $J(B)$ in (32). Starting with an initial value $B_{0}$, the value of $B$ for the $n$th iteration is expressed as

$$
B_{n}=B_{n-1}-\frac{J\left(B_{n-1}\right)}{\left.\frac{\partial J(B)}{\partial B}\right|_{B=B_{n-1}}} .
$$

We stop the iteration at $n=n_{s}$, say, when the relative error $\left|\left(B_{n_{s}}-B_{n_{s}-1}\right) / B_{n_{s}}\right|$ is within a target value, and put $B_{\text {subopt }}=B_{n_{s}}$.

Alternatively, the Gaussian quadrature rule (GQR) can be efficiently applied to the evaluation of the integrals in (32), since they involve averaging of a function over a random variable whose p.d.f. is unknown. The calculation of GQR provides a set of weights and abscissas [12, eq. (25.4.45); p. 923, table (25.9)] such that the approximation

$$
\int_{a}^{b} G(x) W(x) d x \approx \sum_{j=1}^{K} w_{j} G\left(x_{j}\right)
$$

is exact if $G(x)$ is a polynomial of degree up to $2 K-1$ [13]. The values of $w_{j}$ and $x_{j}$ depend on the weight function and the integration interval, and can be computed by finding a set of orthogonal polynomials over $W(x)$ on $[a, b]$. From the algorithm proposed in [14], if $W(x)$ is the p.d.f. $f_{\gamma}(x)$ of the random variable $\gamma$, the $K$-point GQR can be computed using the first $2 K-1$ moments of $\gamma$. All the moments of $\gamma$ can be found in closed-form in [5].

The application of GQR produces the following analytical expression for (32)

$$
\begin{aligned}
\sum_{j=1}^{K} & w_{j}\left\{\sum_{i=\frac{M}{2}}^{M-2}\{-(2 i-M)\right. \\
& \times e^{-g\left((2 i-M+1) \sqrt{x_{j}}-(2 i-M) B\right)^{2}} \\
+ & (2 i-M+2) \\
& \left.\times e^{-g\left((2 i-M+2) B-(2 i-M+1) \sqrt{x_{j}}\right)^{2}}\right\} \\
- & \left.(M-2) e^{-g\left((M-1) \sqrt{x_{j}}-(M-2) B\right)^{2}}\right\} \\
= & 0 .
\end{aligned}
$$

Note that for accuracy at the second significant digit of $B$, only the first 11 moments $(K=6)$ are required.

\section{A. Case of Large $L$}

When $L$ is large, that is, $L \gg 1$, and the fading gain magnitudes $\alpha_{1}, \ldots, \alpha_{L}$ are independent (which implies that the instantaneous branch SNRs $\gamma_{1}, \ldots, \gamma_{L}$ are also independent), using the central limit theorem, we can say that $\sqrt{\gamma}$, given by

$$
\sqrt{\gamma}=\frac{1}{\sqrt{L}} \sum_{l=1}^{L} \sqrt{\gamma_{k}},
$$

approximates to a Gaussian distribution of mean, say, $\mu$ and variance, say, $\sigma^{2}$. From (35) and the independence of $\gamma_{1}, \ldots, \gamma_{L}, \mu$ and $\sigma^{2}$ are

$$
\begin{aligned}
\mu & =\frac{1}{\sqrt{L}} \sum_{l=1}^{L} \mathbf{E}\left[\sqrt{\gamma_{k}}\right], \\
\sigma^{2} & =\frac{1}{L} \sum_{l=1}^{L} \operatorname{var}\left[\sqrt{\gamma_{k}}\right], \quad \sigma>0,
\end{aligned}
$$

where $\mathbf{E}[\cdot]$ denotes the expectation and $\operatorname{var}[\cdot]$ the variance. 
Averaging (29) over the p.d.f. of $\sqrt{\gamma}$, we get

$$
\begin{gathered}
P_{e}(B, M)=\frac{2}{M} \sum_{i=\frac{M}{2}}^{M-2}\left\{\int_{0}^{\infty} Q(\sqrt{2 g}[(2 i-M+1) x\right. \\
+\int_{0}^{\infty} Q(\sqrt{2 g}[(2 i-M+2) B \\
\left.\left.+\int_{0}^{\infty} Q(\sqrt{2 g}[(M-1) x]) f_{\sqrt{\gamma}(x) d x}-(2 i-M+1) x\right] f_{\sqrt{\gamma}}(x) d x\right\} \\
-(M-2) B]) f_{\sqrt{\gamma}(x) d x] .}
\end{gathered}
$$

Substituting

$$
f_{\sqrt{\gamma}}(x) \approx \frac{1}{\sqrt{2 \pi} \sigma} e^{-\frac{1}{2}\left(\frac{x-\mu}{\sigma}\right)^{2}}, \quad-\infty<x<\infty
$$

in (37), and changing the variable $x$ to $y=(x-\mu) / \sigma$, we can rewrite (37) approximately as

$$
\begin{aligned}
P_{e}(B, M) \approx \frac{2}{M}[ & \sum_{i=\frac{M}{2}}^{M-2}\left\{\int_{-\infty}^{\infty} Q(\sqrt{2 g}[(2 i-M+1) \sigma y\right. \\
& +(2 i-M+1) \mu-(2 i-M) B]) \\
& \times \frac{1}{\sqrt{2 \pi}} e^{-\frac{y^{2}}{2}} d y \\
+ & \int_{-\infty}^{\infty} Q(\sqrt{2 g}[(2 i-M+2) B \\
& -(2 i-M+1) \mu-(2 i-M+1) \sigma y]) \\
& \left.\times \frac{1}{\sqrt{2 \pi}} e^{-\frac{y^{2}}{2}} d y\right\} \\
+ & \int_{-\infty}^{\infty} Q(\sqrt{2 g}[(M-1) \sigma y \\
& +(M-1) \mu-(M-2) B]) \\
& \left.\times \frac{1}{\sqrt{2 \pi}} e^{-\frac{y^{2}}{2}} d y\right] .
\end{aligned}
$$

Using the result

$$
\int_{-\infty}^{\infty} Q(C y+D) \frac{1}{\sqrt{2 \pi}} e^{-\frac{y^{2}}{2}} d y=Q\left(\frac{D}{\sqrt{1+C^{2}}}\right)
$$

in (39), we obtain

$$
\begin{aligned}
& P_{e}(B, M) \\
& \approx \frac{2}{M}\left[\sum _ { i = \frac { M } { 2 } } ^ { M - 2 } \left\{Q\left(\frac{\sqrt{2 g}[(2 i-M+1) \mu-(2 i-M) B]}{\sqrt{1+2 g(2 i-M+1)^{2} \sigma^{2}}}\right)\right.\right. \\
& \left.+Q\left(\frac{\sqrt{2 g}[(2 i-M+2) B-(2 i-M+1) \mu]}{\sqrt{1+2 g(2 i-M+1)^{2} \sigma^{2}}}\right)\right\}
\end{aligned}
$$

$$
\left.+Q\left(\frac{\sqrt{2 g}[(M-1) \mu-(M-2) B]}{\sqrt{1+2 g(M-1)^{2} \sigma^{2}}}\right)\right] .
$$

To obtain $B_{\text {subopt }}$ for large $L$, we solve for $B$ in the equation obtained by putting the partial derivative of $P_{e}(B, M)$ in (41) with respect to $B$ to zero. In other words, $B_{\text {subopt }}$ is a solution of the closed-form equation

$$
\begin{gathered}
\sum_{i=\frac{M}{2}}^{M-2} \frac{1}{\sqrt{1+2 g(2 i-M+1)^{2} \sigma^{2}}}\{-(2 i-M) \\
\times e^{-\frac{g[(2 i-M+1) \mu-(2 i-M) B]^{2}}{\left[1+2 g(2 i-M+1)^{2} \sigma^{2}\right]}} \\
+(2 i-M+2) \\
\left.\times e^{-\frac{g[(2 i-M+2) B-(2 i-M+1) \mu]^{2}}{\left[1+2 g(2 i-M+1)^{2} \sigma^{2}\right]}}\right\} \\
-\frac{(M-2)}{\sqrt{1+2 g(M-1)^{2} \sigma^{2}}} \\
\times e^{-\frac{g[(M-1) \mu-(M-2) B]^{2}}{\left[1+2 g(M-1)^{2} \sigma^{2}\right]}}=0 .
\end{gathered}
$$

To obtain an approximate closed-form expression for $B_{\text {subopt }}$ for large $L$ and large SNR, consider the case of independent and identically distributed (i.i.d.) Rayleigh fading, when $\gamma_{1}, \ldots, \gamma_{L}$ are i.i.d. exponential random variables, with

$$
\mathbf{E}\left[\gamma_{k}\right]=\Gamma, \quad \mathbf{E}\left[\sqrt{\gamma_{k}}\right]=\sqrt{\frac{\pi}{4} \Gamma} .
$$

Substituting (43) in (36), we get

$$
\mu^{2}=\frac{L \pi}{4} \Gamma, \quad \sigma^{2}=\left(\frac{4-\pi}{4}\right) \Gamma, \quad \frac{\mu^{2}}{\sigma^{2}}=\frac{L \pi}{4-\pi},
$$

where $\Gamma$ represents the average SNR per diversity branch.

For large SNR, $\Gamma$ is large, implying

$$
\mu^{2} \gg \sigma^{2} \gg 1
$$

when $L$ is also large. Under this condition, only the $i=M / 2$ term on the left-hand side of (42) is significant. Considering only this term, we obtain from (42) the approximate quadratic equation

$$
B^{2}-\frac{2(M-1)}{(3 M-4)} \mu B-\frac{2(M-1)^{2}}{M(3 M-4)} \sigma^{2} \ln \left(\frac{2 M-2}{M-2}\right) \approx 0 .
$$

Solving (46), we get

$$
\begin{aligned}
B_{\text {subopt }} & \approx \frac{2(M-1)}{(3 M-4)} \mu\left[1+\frac{(3 M-4)}{2 M} \frac{\sigma^{2}}{\mu^{2}} \ln \left(\frac{2 M-2}{M-2}\right)\right] \\
& \approx \frac{2(M-1)}{(3 M-4)} \mu .
\end{aligned}
$$

It is clear from (47) and (44) that $B_{\text {subopt }}$ increases with increase of $L$ for large SNR $\Gamma$. It decreases with increase of $M$, going from $3 \mu / 4$ at $M=4$ to $2 \mu / 3$ for large $M$.

\section{CASE OF $M$-QAM}

When $M$-QAM is used with a square constellation, that is, with $M$ being the square of an even number, the constellation can be viewed as two $\sqrt{M}$-PAM constellations in quadrature, each having half the average signal energy. As a result, the 

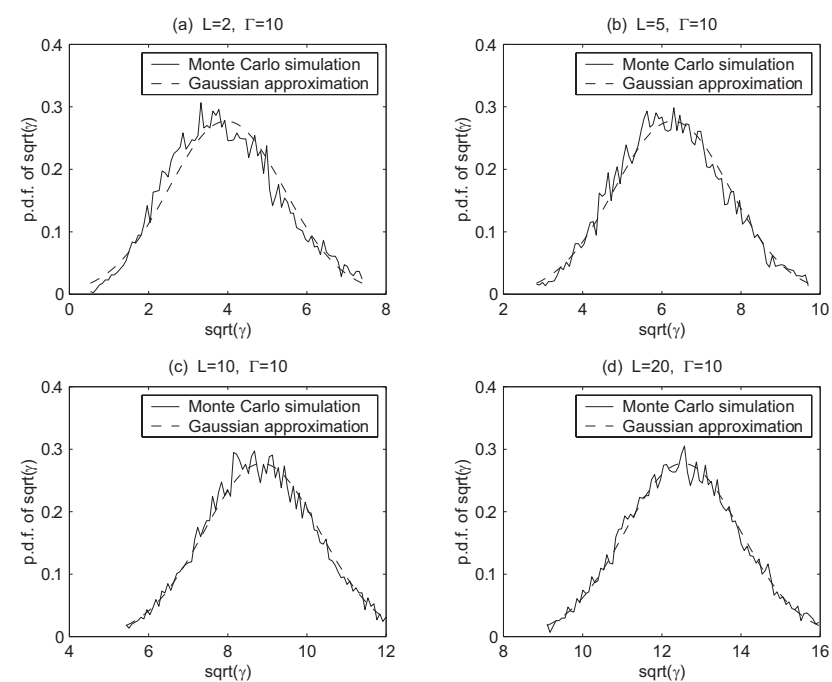

Fig. 1. Comparison of the p.d.f. of $\gamma^{1 / 2}$ generated by Monte Carlo simulation in case of i.i.d. Rayleigh fading with that from the Gaussian approximation, for average SNR per branch $\Gamma=10$ and different values of number of branches $L$.

SEP can be easily expressed in terms of the SEP of $\sqrt{M}$ PAM. If $\left(P_{e}(\gamma, B, M)\right)_{Q A M}$ denotes the SEP of the $M$-QAM constellation as a function of instantaneous combiner output SNR $\gamma$, normalized bias $B$, and constellation size $M$, then

$$
\begin{aligned}
\left(P_{e}(\gamma, B, M)\right)_{Q A M}= & 2 P_{e}(\gamma / 2, B / \sqrt{2}, \sqrt{M}) \\
& -P_{e}^{2}(\gamma / 2, B / \sqrt{2}, \sqrt{M}),
\end{aligned}
$$

where $P_{e}(\gamma / 2, B / \sqrt{2}, \sqrt{M})$ is the SEP of $\sqrt{M}$-PAM evaluated at instantaneous combiner output SNR $\gamma / 2$ and normalized bias $B / \sqrt{2}$ (because the PAM constellation has half the average signal energy of the QAM constellation).

To obtain the suboptimal bias, we need to minimize $\left(P_{e}(\gamma, B, M)\right)_{Q A M}$ with respect to $B$ and average over the statistics of $\gamma$. Differentiating with respect to $B$, we get

$$
\begin{aligned}
& \frac{\partial}{\partial B}\left(P_{e}(\gamma, B, M)\right)_{Q A M} \\
& =2\left(1-P_{e}(\gamma / 2, B / \sqrt{2}, \sqrt{M})\right) \\
& \quad \times \frac{\partial}{\partial B} P_{e}(\gamma / 2, B / \sqrt{2}, \sqrt{M}) .
\end{aligned}
$$

Let $B_{\text {subopt }}\left(\mu, \sigma^{2}, M\right)$ represent the bias in case of $M$-PAM for large $L$. Putting the derivative (49) to zero and averaging over the statistics of $\gamma$, we get a bias which can be written as

$$
\left(B_{\text {subopt }}\left(\mu, \sigma^{2}, M\right)\right)_{Q A M}=\sqrt{2} B_{\text {subopt }}\left(\frac{\mu}{\sqrt{2}}, \frac{\sigma^{2}}{2}, \sqrt{M}\right)_{(50)} \text {. }
$$

Note that when we apply the Gaussian approximation in case of $M$-QAM, it is not possible to obtain a closed-form approximate expression for $P_{e}(B, M)$ as in (41) owing to the difficulty of averaging the $P_{e}^{2}(\cdot)$ term of (48) over $\gamma$.

\section{NuMERICAL RESULTS}

In this section we present selected numerical results, obtained by Monte Carlo simulation of the instantaneous combiner output SNR $\gamma$ as well as by Gaussian approximation of the p.d.f. of $\sqrt{\gamma}$. In Fig. 1 Monte Carlo simulation results

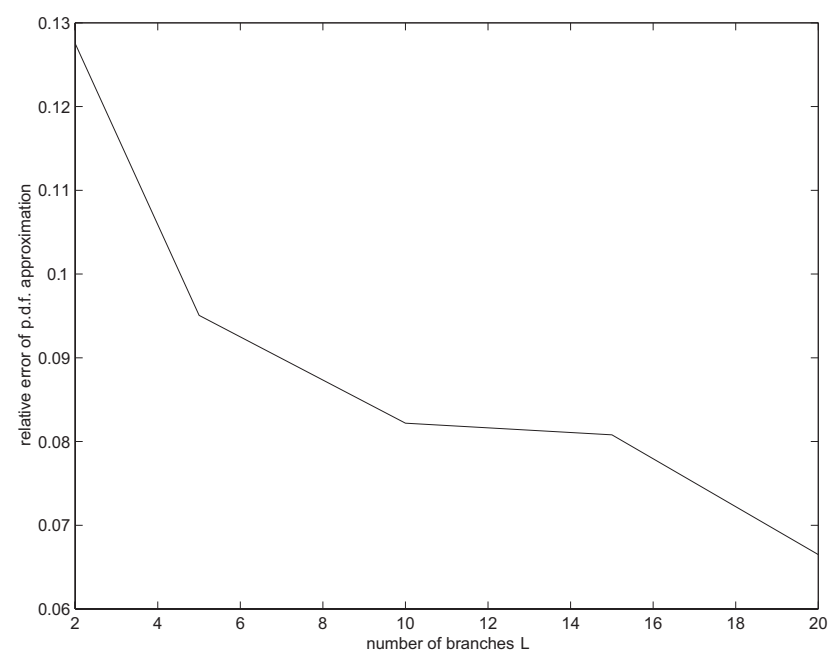

Fig. 2. Relative error of p.d.f. approximation versus number of branches $L$ in case of i.i.d. Rayleigh fading with average SNR per branch $\Gamma=10$.

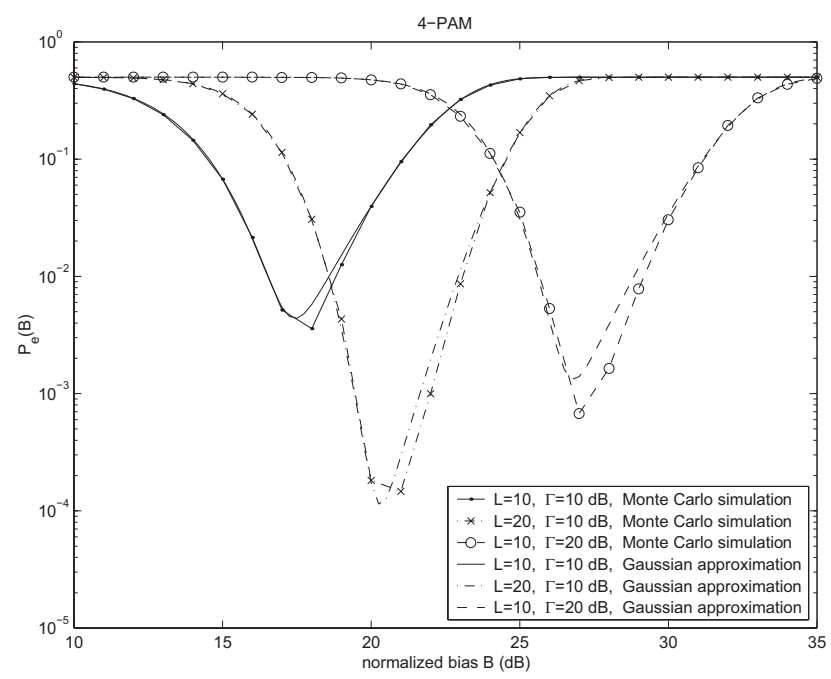

Fig. 3. Comparison of the SEP $P_{e}(B)$ computed by Monte Carlo simulation in case of i.i.d. Rayleigh fading with that from the Gaussian approximation, for 4-PAM with different values of number of branches $L$ and average SNR per branch $\Gamma$.

are shown to compare the p.d.f. of the sum of i.i.d Rayleigh random variables with that of the Gaussian approximation. An excellent match is observed, especially for large values of $L$. Moreover, in Fig. 2 the relative error of this approximation is depicted as a function of the number of branches $L$, also in the case of i.i.d Rayleigh fading. Furthermore, in order to show the impact of this approximation in our analysis, we compare in Fig. 3 the SEP $P_{e}(B)$ for 4-PAM, as evaluated by Monte Carlo simulation, with that from the Gaussian approximation. We see a good agreement between the simulation and approximation results. We also find that the suboptimal bias shifts to the right as $L$ or the average SNR per diversity branch $\Gamma$ increases, which is in agreement with the approximate expression for the normalized suboptimal bias $B_{\text {subopt }}$ in (47). In Fig. 4, $P_{e}(B)$, obtained by Monte Carlo simulation, is plotted versus the normalized bias $B$ for 16-QAM for several values of $L$ and $\Gamma$. The same trend as in Fig. 3 is observed when $L$ and $\Gamma$ vary. 


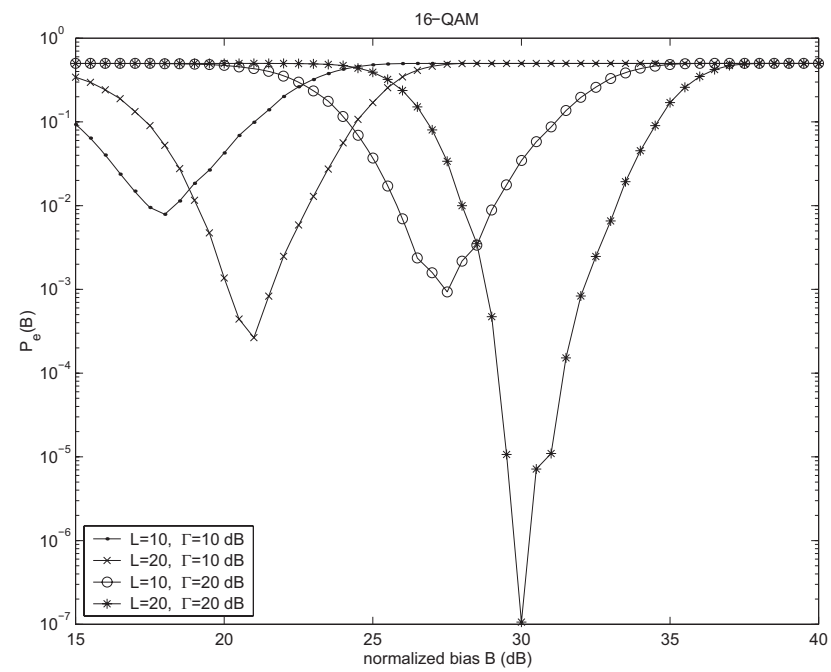

Fig. 4. SEP $P_{e}(B)$ computed by Monte Carlo simulation versus normalized bias $B$ in case of i.i.d. Rayleigh fading for 16-QAM with different values of $L$ and $\Gamma$.

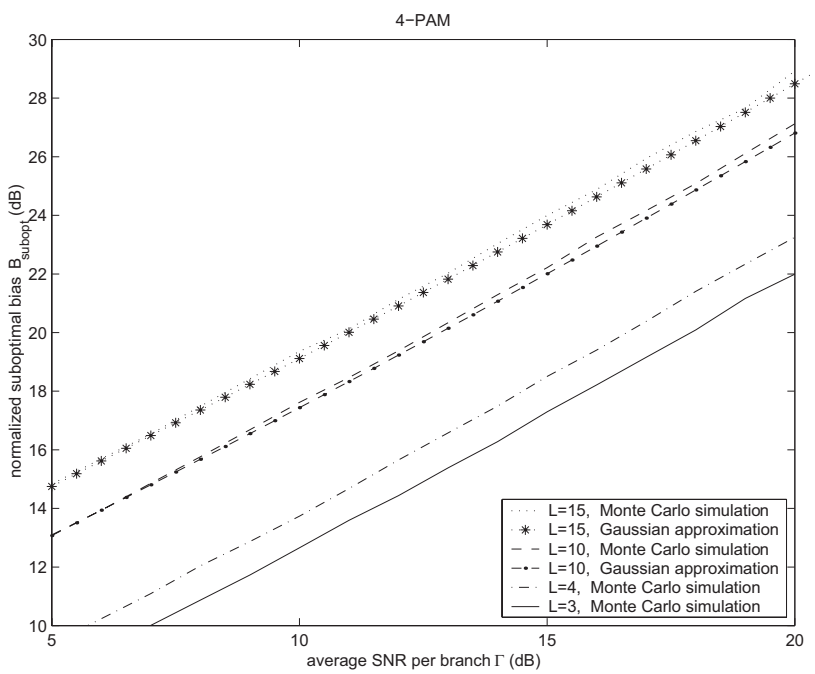

Fig. 5. Normalized suboptimal bias $B_{\text {subopt }}$ versus $\Gamma$ in case of i.i.d. Rayleigh fading for 4-PAM with different values of $L$.

Fig. 5 shows the variation of the normalized suboptimal bias $B_{\text {subopt }}$ in case of i.i.d. Rayleigh fading, computed using Newton-Raphson method having a target relative error of $10^{-3}$, with $\Gamma$ and $L$, for 4 -PAM. To obtain the Monte Carlo simulation results for $L=3,4,10,15$, we solve the equation (32), whereas to obtain the Gaussian approximation results for $L=10,15$, we solve the equation (42). A good agreement is seen between the simulation and approximation results for $L=10,15$. In Fig. 6 the minimized error probability $P_{e}\left(B_{\text {subopt }}\right)$ of 4-PAM, which is obtained after the evaluation of the suboptimal value for $B$, is plotted versus $\Gamma$ for different values of $L$. For $L=5$, both Monte Carlo simulation and Gaussian approximation results are shown. We find that although $P_{e}\left(B_{\text {subopt }}\right)$ decreases with increase of $L$, it tends to saturate as $\Gamma$ increases.

\section{CONCLUSION}

An analytical method to compute suboptimal values for the bias in EGC receivers with unequal energy constellations

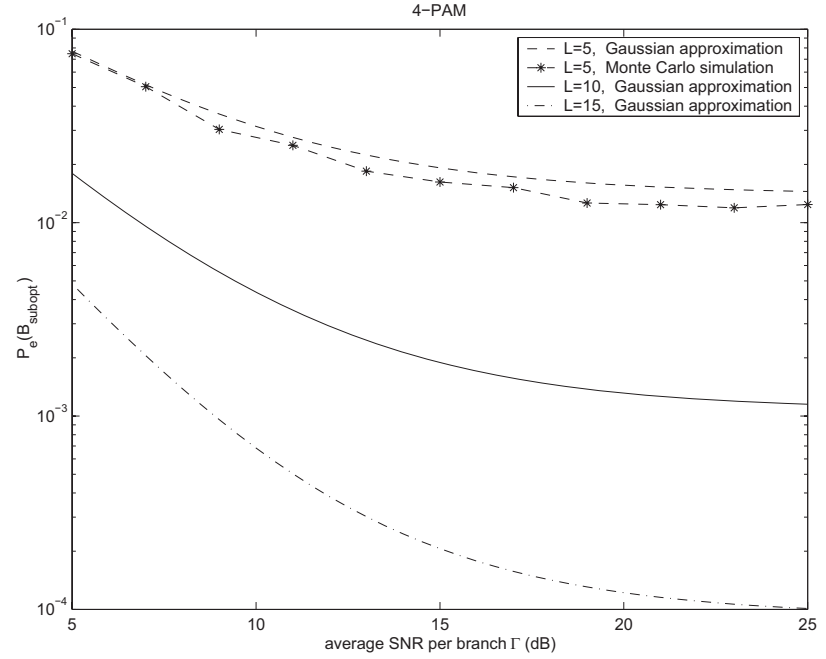

Fig. 6. Minimum SEP $P_{e}\left(B_{\text {subopt }}\right)$ versus $\Gamma$ in case of i.i.d. Rayleigh fading for 4-PAM with different values of $L$.

( $M$-PAM and $M$-QAM) has been presented. The proposed suboptimal bias minimizes the SEP, and depends on the statistics of the fading gains and not on their instantaneous values and can be evaluated by solving numerically an exact single integral equation. It has been verified via Monte Carlo simulation that for the case of large number of branches, the proposed closed-form equation for the computation of the suboptimal bias provides adequate accuracy.

\section{REFERENCES}

[1] N. C. Beaulieu and A. A. Abu-Dayya, "Analysis of equal gain diversity on Nakagami fading channels," IEEE Trans. Commun., vol. 39, no. 2, pp. 225-234, Feb. 1991

[2] Q. T. Zhang, "A simple approach to probability of error for equal gain combiners over Rayleigh channels," IEEE Trans. Veh. Technol., vol. 48, no. 4, pp. 1151-1154, July 1999.

[3] A. Annamalai, C. Tellambura, and V. K. Bhargava, "Equal-gain diversity receiver performance in wireless channels," IEEE Trans. Commun., vol. 48, no. 10 , pp. 1732-1745, Oct. 2000.

[4] G. K. Karagiannidis, D. A. Zogas, and S. A. Kotsopoulos, "Properties of the EGC output SNR over correlated Nakagami- $m$ fading channels," IEEE Trans. Wireless Commun., vol. 3, no. 5, pp. 1764-1769, Sep. 2004.

[5] G. K. Karagiannidis, "Moments-based approach to the performance analysis of equal-gain diversity in Nakagami- $m$ fading," IEEE Trans. Commun., vol. 52, no. 5, pp. 685-690, May 2004.

[6] R. K. Mallik, M. Z. Win, and J. H. Winters, "Performance of dualdiversity predetection EGC in correlated Rayleigh fading with unequal branch SNRs," IEEE Trans. Commun., vol. 50, no. 7, pp. 1041-1044, July 2002.

[7] G. K. Karagiannidis, D. A. Zogas, and S. A. Kotsopoulos, "BER performance of dual predetection EGC in correlative Nakagami- $m$ fading," IEEE Trans. Commun., vol. 52, no. 1, pp. 50-53, Jan. 2004.

[8] M. K. Simon and M.-S. Alouini, Digital Communication over Fading Channels, 2nd ed. New York: Wiley, 2004.

[9] N. C. Beaulieu, "An infinite series for the computation of the complementary probability distribution function of a sum of independent random variables and its application to the sum of Rayleigh random variables," IEEE Trans. Commun., vol. 38, no. 10, pp. 1463-1474, Sep. 1990.

[10] S. W. Halpern,"The effect of having un-equal branch gains in practical pre-detection diversity systems for mobile radio," IEEE Trans. Veh. Technol., vol. 26, pp. 94-105, Feb. 1977.

[11] C. Wylie and L. Barrett, Advanced Engineering Mathematics. New York: McGraw-Hill, 1982.

[12] M. Abramowitz and I. A. Stegun, eds., Handbook of Mathematical Functions, 9th printing. New York: Dover, 1970.

[13] W. Gautschi, "Orthogonal polynomials and quadrature," Elec. Trans. Numer. Anal., vol. 9, pp. 65-76, 1999. 
[14] G. H. Golub and J. H. Welsh, "Calculation of Gauss quadrature rules," Math. Comput., vol. 23, pp. 221-230, Apr. 1969.

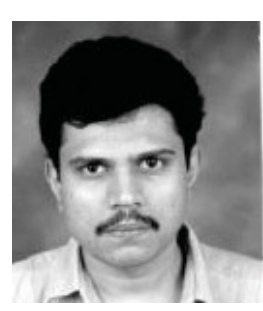

Ranjan K. Mallik (S'88-M'93-SM'02) received the B.Tech. degree from the Indian Institute of Technology, Kanpur, in 1987 and the M.S. and Ph.D. degrees from the University of Southern California, Los Angeles, in 1988 and 1992, respectively, all in Electrical Engineering. From August 1992 to November 1994, he was a scientist at the Defence Electronics Research Laboratory, Hyderabad, India, working on missile and EW projects. From November 1994 to January 1996, he was a faculty member of the Department of Electronics and Electrical Communication Engineering, Indian Institute of Technology, Kharagpur. In January 1996, he joined the faculty of the Department of Electronics and Communication Engineering, Indian Institute of Technology, Guwahati, where he worked till December 1998. Since December 1998, he has been with the Department of Electrical Engineering, Indian Institute of Technology, Delhi, where he is a Professor. His research interests are in communication theory and systems, difference equations, and linear algebra.

Dr. Mallik is a member of Eta Kappa Nu. He is also a member of the IEEE Communications and Information Theory Societies, the American Mathematical Society, the International Linear Algebra Society, and The Institution of Engineering and Technology, a fellow of the Indian National Academy of Engineering and The Institution of Electronics and Telecommunication Engineers, a life member of the Indian Society for Technical Education, and an associate member of The Institution of Engineers (India). He is an Editor for the IEEE TRANSACTIONS ON WIRELESS COMMUNICATIONS.

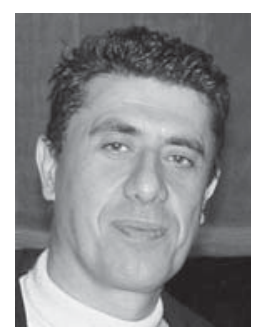

George K. Karagiannidis (S'95-M'97-SM'04) received his university degree in 1987 and his Ph.D. degree in 1999, both in Electrical Engineering, from the University of Patras, Patras, Greece. From 2000 to 2004, he was a Researcher at the Institute for Space Applications and Remote Sensing, National Observatory of Athens, Greece. In June 2004, he joined the faculty of Aristotle University of Thessaloniki, Greece, where he is currently an Assistant Professor at the Department of Electrical and Computer Engineering.

His major research interests include wireless communications theory, digital communications over fading channels, satellite communications, and freespace optical communications. He has published and presented more than 70 technical papers in scientific journals and international conferences; he is co-author in two chapters in books and also co-author in a Greek edition book on mobile communications. He is a member of the editorial boards of the IEEE TRANSACTIONS ON COMMUNICATIONS, the IEEE COMMUNICATIONS LETTERS, and the EURASIP Journal on Wireless Comnmunications and Networking. 